\title{
A Two-Stage Robust Optimization Method Based on the Expected Scenario for Islanded Microgrid Energy Management
}

\author{
Qing Duan, Wanxing Sheng, Haoqing Wang ${ }^{(D}$, Caihong Zhao, and Chunyan Ma \\ Power Distribution Technology Center, China Electric Power Research Institute, Beijing 100192, China \\ Correspondence should be addressed to Haoqing Wang; wangbecks@163.com
}

Received 7 June 2021; Revised 18 June 2021; Accepted 5 August 2021; Published 4 October 2021

Academic Editor: Shi Cheng

Copyright (C) 2021 Qing Duan et al. This is an open access article distributed under the Creative Commons Attribution License, which permits unrestricted use, distribution, and reproduction in any medium, provided the original work is properly cited.

\begin{abstract}
One of the main challenges in microgrid system energy management is dealing with uncertainties such as the power output from renewable energy sources. The classic two-stage robust optimization (C-TSRO) method was proposed to cope with these uncertainties. However, this method is oriented to the worst-case scenario and is therefore somewhat conservative. In this study, focusing on the energy management of a typical islanded microgrid and considering uncertainties such as the power output of renewable energy sources and the power demand of loads, an expected-scenario-oriented two-stage robust optimization (ETSRO) method is proposed to alleviate the conservative tendency of the C-TSRO method because the E-TSRO method chooses to optimize the system cost according to the expected scenario instead, while ensuring the feasibility of the first-stage variables for all possible scenarios, including the worst case. According to the structural characteristics of the proposed model based on the E-TSRO method, a column-and-constraint generation (C \& CG) algorithm is utilized to solve the proposed model. Finally, the effectiveness of the E-TSRO model and the solution algorithm are analysed and validated through a series of experiments, thus obtaining some important conclusions, i.e., the economic efficiency of system operation can be improved at about $6.7 \%$ in comparison with the C-TSRO results.
\end{abstract}

\section{Introduction}

Microgrids are small power generation and distribution systems composed of distributed power supplies, energy storage systems (ESSs), user loads, energy conversion devices, and protective devices. They are important for the effective utilization of renewable energy resources and to improve the reliability of the energy supply under various loads. Microgrids can be classified into two types, namely, islanded and grid-connected, based on whether or not they connect to the power grid. Islanded microgrids are primarily used to supply power to remote regions that have yet to be reached by the power grid (e.g., islands). Grid-connected microgrids are connected to the power grid, through which energy can be traded based on the difference between the peak and off-peak electricity prices. In the event of a large power grid malfunction, a grid-connected microgrid can switch to the islanding mode.
Energy management is an important part of the scheduling and operational management of a microgrid, wherein scheduling decisions are made based on the behaviour of its components (e.g., the power generators, ESS, and response loads) to ensure a stable and efficient operation. Various uncertainties such as the power output from renewable energy sources (e.g., wind and solar energy) and loads pose significant challenges in microgrid energy management, and their effective treatment has become an important research topic in this domain. Accordingly, researchers have proposed two main types of uncertainty modelling methods for microgrid energy management, namely, stochastic programming and robust optimization [1].

In the stochastic programming method, the probability distribution of an uncertain parameter is assumed to be known in advance. This probability distribution is then used to transform an uncertainty problem to a deterministic one. 
Researchers [2-6] have developed stochastic programming models for the energy management of grid-connected microgrids to minimize the overall cost under uncertainties in single or multiple factors (e.g., electricity transaction price, power output from renewable energy sources, and loads). These models differ mainly in the composition of the target cost, the uncertain factors considered, and the scenario generation method. Other researchers [7-10] have developed models for grid-connected microgrids with optimal energy management objectives that are not limited to minimization of the overall cost. The energy management models developed by Tabar et al. [8] and Gazijahani et al. [10] use multiple optimization objectives. The model built by Tabar et al. [8] has two optimization objectives, namely, to minimize the operational cost and the level of air pollution, whereas those in the model developed by Gazijahani et al. [10] aim to minimize the operational cost and to maximize the power supply reliability. The optimization objectives of the stochastic programming energy management model developed by $\mathrm{Hu}$ et al. [9] are divided into two stages: reduction of the investment cost of the microgrid in the first stage and minimization of the operational and management cost of the microgrid in the second stage. In addition to these studies on grid-connected microgrids, studies have also been conducted on islanded microgrids [11-13]. Cau et al. [11] investigated an islanded microgrid with a hydrogen production unit and hydrogen fuel cells as well as renewable (wind and solar) energy sources and energy storage batteries; the optimization objective of the proposed energy management model was to maximize the utilization of the renewable energy sources. Rezaei and Kalantar [12] considered the frequency control problem of islanded microgrids and developed a microgrid energy management model that minimized the deviation from the day-ahead frequency. Farzin et al. [13] optimized the expected operational and management cost of a microgrid during an islanding event. In addition to scenario-probability-based stochastic programming, researchers [14] have used chance constraintbased stochastic programming to handle uncertain factors in microgrid energy management. The main difficulty in applying stochastic programming-based microgrid energy management is the accurate determination of the multiple scenarios used to describe uncertainties, particularly the probability of occurrence of each scenario. Moreover, the complexity of the model solution scheme increases with the number of scenarios used to represent the uncertainties [15].

The robust optimization method does not require a priori knowledge of the probability distribution for an uncertain parameter. Instead, the fluctuation range of an uncertain parameter is represented by a specified interval, and a feasible solution can be guaranteed with a high probability as long as the realized value of the uncertain parameter lies in the given interval [16]. According to the stage division of decision variables, available studies on microgrid energy management based on robust optimization can be divided into two types, namely, those based on single-stage robust optimization [17-21] and those based on multistage robust optimization. In the former, all the decision variables for microgrid energy management are determined at the same time. For example, Kuznetsova et al. [20] and Zhang et al. [21] investigated microgrids that only contain electrical power whereas Wang et al. [17] and Luo et al. [18] studied microgrids that consist of CHP (combined heat and power) and CCHP (combined cooling, heating, and power), respectively. Microgrid energy management methods based on single-stage robust optimization offer the advantage of relatively simple models that can be solved easily, but these models require decision-makers to make decisions in parallel before knowing the realized values of the uncertain parameters. Typically, there are equality constraints (i.e., energy supply and demand should be balanced at all times) in microgrid energy management models, which leads to conservative decisions and is inconsistent with the fact that decisions are often made sequentially in stages in engineering practice. In contrast, in multistage optimization such as two-stage robust optimization [22-25], some decisions (e.g., the on/off status of the generators, the charge/discharge status of the ESS, the transaction status, or preagreed quantity of power transacted with the power grid) are made before knowing the realized values of the uncertain parameters whereas the remaining variables are determined after the values of the uncertain parameters have been determined. Therefore, this approach can be used to deal with equality constraints in microgrid energy management models. To cope with the min-max-min optimization objectives embedded in two-stage robust optimization models, researchers typically adopt the columnand-constraint generation (C \& CG) algorithm [26] to solve these models [23-25] but other algorithms have also been proposed. For example, the Lyapunov optimization algorithm (Hu et al. [27]) has been utilized to yield approximate solutions. In addition, Xiang et al. [22] established a scenario-robust-combined two-stage robust optimization model for energy management and proposed an orthogonal array-based search algorithm to solve the model. For an isolated microgrid on Nanji Island in China that contains a seawater pumped storage system, Tian et al. [28] established a multitime-scale islanded microgrid energy management model based on robust optimization and developed a Bender's decomposition and cutting plane algorithm to solve the proposed model. Zhang et al. [29] established a two-stage robust optimization model for multimicrogrid energy management, which was solved using the C \& CG algorithm. Hussain et al. [30] studied grid resilience by investigating possible outages to ensure optimal operation and scheduling of critical loads. In the aforementioned studies, the decision variables were determined using the classic two-stage robust optimization (C-TSRO) method that is oriented to the worst-case scenario. However, in practice, the probability of the worst-case scenario occurring is very low, making the solutions obtained using the C-TSRO method relatively conservative, especially as continuous improvement by an interval prediction algorithm often decreases the difference between the actual and predicted expected values of the uncertain parameters. As a result, the solution obtained based on the worst-case scenario is always worse than that based on the expected scenario considering the optimization objectives (i.e., economic efficiency) [31]. 
Targeting on the drawback in adopting the C-TSRO method, an expected-scenario-oriented two-stage robust optimization (E-TSRO) model is proposed for microgrid energy management of typical islanded microgrid systems composed of renewable energy equipment (wind and solar), ESSs, diesel generators, and various types of loads. The model accounts for uncertainties in factors such as the power output from renewable energy sources and the load demand. There are two stages in the E-TSRO model for determining the decision variables of microgrid energy management, namely, prescheduling (day-ahead) and rescheduling (intraday). Compared to the C-TSRO method oriented to the worst-case scenario, the E-TSRO method primarily focuses on the optimization function (i.e., maximizing the economic efficiency of the system) based on the expected scenario, while ensuring the feasibility of solutions under any realized scenario. The E-TSRO method ensures that there are always feasible rescheduled solutions for these second-stage decision variables under any realized scenario based on the predetermined values of the first-stage decision variables, resulting in less conservative decisions. Thus, safe and stable microgrid operation can be achieved in uncertain environments while maintaining a relatively high economic efficiency. Taking into consideration the nonlinear formulation of the proposed model and the complexity of solving this type of model, the model is equivalently transformed and a C \& CG algorithm is developed to solve the transformed model. Experiments are used to validate the advantages of the E-TSRO model and the effectiveness of the developed algorithm.

The main contributions of this paper are summarized as follows.

(1) Instead of orienting to the worst case, an expectedscenario-oriented two-stage robust optimization (ETSRO) method is proposed to alleviate the conservative tendency of the classic two-stage robust optimization (C-TSRO) method.

(2) According to the structural characteristics of the proposed model based on the E-TSRO method, a column-and-constraint generation (C \& CG) algorithm is utilized to solve the proposed model.

This paper is organized as follows. In Section 1, the research background and literature review are presented. Section 2 provides a basic model for islanded microgrid energy management. In order to solve the proposed model, the E-TSRO framework and its transformation along with the C \& CG solution algorithm are developed in Section 3. Section 4 depicts the experimental validation process and analytical results. Finally, summary and future work are concluded in Section 5.

\section{Basic Model for Islanded Microgrid Energy Management}

The islanded microgrid investigated in this study primarily consists of wind and photovoltaic (PV) power generators, loads (including critical and curtailable loads), one ESS, and distributed generators (DGs). The notations used in this study and their corresponding abbreviations are listed as follows.

2.1. Notation Description. Tables 1-3 depict system parameters, predicted parameters, and decision variables, respectively, that are used in the model.

The main constraints and optimization objectives of the basic model for islanded microgrid energy management are described below.

\subsection{Main Constraints of the Basic Model}

2.2.1. DG Constraints. In this study, DGs primarily refer to controllable generators and are represented by diesel generators. For a DG, constraints are placed on the maximum ramp power, the maximum and minimum operating power, and the minimum duration before startup and shutdown.

(1) Generation power and ramp power constraints:

$$
\begin{aligned}
-\Delta P_{\mathrm{DG}, i} \Delta t & \leq P_{\mathrm{DG}, i}(k)-P_{\mathrm{DG}, i}(k-1) \\
& \leq \Delta P_{\mathrm{DG}, i} \Delta t, \quad \forall i, \forall k, \\
\delta_{\mathrm{DG}, i}(k) P_{\mathrm{DG}, i}^{\min } & \leq P_{\mathrm{DG}, i}(k) \\
& \leq \delta_{\mathrm{DG}, i}(k) P_{\mathrm{DG}, i}^{\max }, \quad \forall i, \forall k .
\end{aligned}
$$

Constraint equations (1) and (2) are the ramp power and generation power constraints on the generator, respectively.

(2) Duration constraints for startup and shutdown:

$$
\begin{aligned}
& \delta_{\mathrm{DG}, i}(k)-\delta_{\mathrm{DG}, i}(k-1) \leq \delta_{\mathrm{DG}, i}\left(\tau_{1}\right), \quad \forall i, \forall k, \tau_{1}=k+1, \ldots, \min \left(k+T_{i}^{\mathrm{up}}-1, T\right), \\
& \delta_{\mathrm{DG}, i}(k-1)-\delta_{\mathrm{DG}, i}(k) \leq 1-\delta_{\mathrm{DG}, i}\left(\tau_{2}\right), \quad \forall i, \forall k, \tau_{2}=k+1, \ldots, \min \left(k+T_{i}^{\text {down }}-1, T\right) . \\
& \chi_{\mathrm{DG}, i}^{\mathrm{up}}(k) \geq c_{\mathrm{DG}, i}^{\mathrm{up}}\left(\delta_{\mathrm{DG}, i}(k)-\delta_{\mathrm{DG}, i}(k-1)\right), \quad \forall i, \forall k, \\
& \chi_{\mathrm{DG}, i}^{\mathrm{down}}(k) \geq c_{\mathrm{DG}, i}^{\mathrm{down}}\left(\delta_{\mathrm{DG}, i}(k-1)-\delta_{\mathrm{DG}, i}(k)\right), \quad \forall i, \forall k,
\end{aligned}
$$

Constraint equation (3) requires that DG $i$ operates for at least $T_{i}^{\text {up }}$ number of periods after startup. Constraint equation (4) requires DG $i$ to remain shut down for at least $T_{i}^{\text {down }}$ number of cycles after shutdown.

(3) Startup and shutdown cost constraints: 
TABLE 1: System parameters.

\begin{tabular}{|c|c|}
\hline$T$ & Number of time intervals or periods in predicted time domain \\
\hline$k$ & Index of time interval $(k \in[1, T])$ \\
\hline$\Delta t$ & Duration of time interval $(\mathrm{h})$ \\
\hline$\beta_{\mathrm{cur}}^{\max }$ and $\beta_{\mathrm{cur}}^{\min }$ & Maximum and minimum allowed curtailable load (\%), respectively \\
\hline$\alpha_{\text {cur }}(k)$ & Penalty weight on curtailment at time interval $k(€ /(\mathrm{kW} \cdot \mathrm{h}))$ \\
\hline$E_{\text {bess }}^{\max }$ and $E_{\text {bess }}^{\min }$ & Maximum and minimum capacity of the ESS $(\mathrm{kW} \cdot \mathrm{h})$, respectively \\
\hline$P_{\text {bess }}^{\max }$ and $P_{\text {bess }}^{\min }$ & Maximum and minimum discharging/charging power of the ESS $(\mathrm{kW})$, respectively \\
\hline$\eta_{\text {bess }}$ & Charging/discharging efficiency (\%) \\
\hline$\varepsilon_{\text {bess }}$ & Self-discharging power of the ESS $(k W)$ \\
\hline $\mathrm{OM}_{\text {bess }}$ & Maintenance cost of the ESS $(€ /(\mathrm{kW} \cdot \mathrm{h}))$ \\
\hline$N_{\mathrm{DG}}$ & Number of DGs \\
\hline$P_{\mathrm{DG}, i}^{\mathrm{max}}$ and $P_{\mathrm{DG}, i}^{\min }$ & Minimum and maximum operating power for DG $i(\mathrm{~kW})$ \\
\hline$T_{i}^{\mathrm{up}, l}$ and $T_{i}^{\mathrm{down}, l}$ & Required minimum durations of DG $i$ before its startup and shutdown (period), respectively \\
\hline$\Delta P_{\mathrm{DG}, i}$ & Maximum ramp power for DG $i(\mathrm{~kW})$ \\
\hline$C_{\mathrm{DG}, i}(k)$ & Fuel cost of controllable DG $i$ at time interval $k(€)$ \\
\hline$a_{i}, b_{i}$, and $c_{i}$ & Quadratic, linear, and constant coefficients of fuel cost function of DG $i$ [32] \\
\hline $\mathrm{OM}_{\mathrm{DG}, i}$ & Maintenance cost for DG $i(€ / \mathrm{h})$ \\
\hline$c_{\mathrm{DG}, i}^{\mathrm{up}}$ and $c_{\mathrm{DG}, i}^{\mathrm{down}}$ & Startup and shutdown costs for DG $i(€)$, respectively \\
\hline
\end{tabular}

TABle 2: Predicted parameters.

$\widetilde{P}_{\text {solar }}(k), \widetilde{P}_{\text {wind }}(k), \widetilde{P}_{\text {cri }}(k)$, and Predicted power of PV generators, wind generators, critical loads, and curtailable loads at time interval $k$ $\widetilde{P}_{\text {cur }}(k) \quad(\mathrm{kW})$, respectively

$\bar{P}_{\text {solar }}(k), \bar{P}_{\text {wind }}(k), \bar{P}_{\text {cri }}(k)$, and Expected value of the predicted power for PV generators, wind generators, critical loads, and curtailable $\bar{P}_{\text {cur }}(k) \quad$ loads during time interval $k(\mathrm{~kW})$, respectively

$\widehat{P}_{\text {solar }}(k), \widehat{P}_{\text {wind }}(k), \widehat{P}_{\text {cri }}(k)$, and Deviation from the expected value of predicted power for PV generators, wind generators, critical loads, $\widehat{P}_{\text {cur }}(k) \quad$ and curtailable loads during time interval $k$, respectively

TABLE 3: Decision variables.

\begin{tabular}{lc}
\hline$\delta_{\mathrm{DG}}(k)$ & On/off status of DG $i$ for time interval $k(1:$ on; $0:$ off $)$ \\
$P_{\mathrm{DG}}(k)$ & Power of DG $i$ for time interval $k(\mathrm{~kW})$ \\
$E_{\text {bess }}(k)$ & Capacity of the ESS at time interval $k(\mathrm{~kW} \cdot \mathrm{h})$ \\
$P_{\text {bess }}(k)$ & Charging/discharging power of the ESS at time interval $k(\mathrm{~kW})$ \\
$\beta_{\text {cur }}(k)$ & Curtailed power percentage of the curtailable loads for time interval $k(\%)$ \\
\hline
\end{tabular}

$$
\chi_{\mathrm{DG}, i}^{\mathrm{up}}(k) \geq 0, \chi_{\mathrm{DG}, i}^{\text {down }}(k) \geq 0
$$

Constraint equations (5) and (6) are methods for calculating the startup and shutdown costs, respectively, for DG $i$ over the time interval $k$, which are identical with [15].

2.2.2. Constraints on the ESS. An ESS plays a vital role in an islanded microgrid and can effectively mitigate asynchronicity between power generation from renewable energy sources and power consumption by users. The main constraints on an ESS are given below:

$$
\begin{gathered}
P_{\text {bess }}^{\min } \leq P_{\text {bess }}(k) \leq P_{\text {bess }}^{\max }, \quad \forall k, \\
E_{\text {bess }}^{\min } \leq E_{\text {bess }}(k) \leq E_{\text {bess }}^{\max }, \quad \forall k, \\
E_{\text {bess }}(k+1)=E_{\text {bess }}(k)+\eta_{\text {bess }} P_{\text {bess }}(k) \Delta t-\varepsilon_{\text {bess }} \Delta t, \quad \forall k .
\end{gathered}
$$

A positive $P_{\text {bess }}(k)$ corresponds to a charged ESS whereas a negative $P_{\text {bess }}(k)$ corresponds to ESS discharge. Constraint equations (8) and (9) show the charge and discharge power limit and capacity limit, respectively. Equation (10) describes the relationship between the ESS capacity and the ESS charge/discharge power. Since the primary focus of this study is the performance of two-stage robust optimization for microgrid energy management based on the expected scenario, to simplify the subsequent dual transformation process of the model, this study does not distinguish the charge power coefficient from the discharge power coefficient, and both coefficients are denoted as $\eta_{\text {bess }}$ in the remainder of this study.

2.2.3. Constraint on Curtailable Load. Two types of loads are mainly considered in the proposed model, namely, critical and curtailable loads. Critical loads are closely related to the basic daily activities of users. As the normal operation of critical loads directly affects the daily life of users, the power demand of these loads (e.g., indoor illumination and 
infrastructure) must be met unconditionally. In contrast, curtailable loads (e.g., air conditioners and ventilation systems) do not have to operate at rated powers, that is, the operating power can be adjusted within a certain range, which is shown below:

$$
\beta_{\text {cur }}^{\min } \leq \beta_{\text {cur }}(k) \leq \beta_{\text {cur }}^{\max }, \quad \forall k .
$$

Moreover, considering that load curtailment affects user comfort, the penalty or compensation cost associated with load curtailment will be considered in calculating the total operational cost.

2.2.4. Microgrid Energy Balance Constraint. Stable and reliable operation at all times is the precondition for optimizing the energy scheduling of an islanded microgrid; therefore, a balance of energy supply and demand must be maintained as follows:

$$
\begin{aligned}
& \widetilde{P}_{\text {cur }}(k)\left(1-\beta_{\text {cur }}(k)\right)+\widetilde{P}_{\text {cri }}(k)+P_{\text {bess }}(k) \\
& =\widetilde{P}_{\text {solar }}(k)+\widetilde{P}_{\text {wind }}(k)+\sum_{i=1}^{N_{\mathrm{DG}}} P_{\mathrm{DG}, i}(k), \quad \forall i, \forall k .
\end{aligned}
$$

2.3. Optimization Objective of the Basic Model. The optimization objective of microgrid energy management is to minimize the total operational cost of the system, which is expressed as

$$
\begin{aligned}
\min F= & \sum_{k=1}^{T} \sum_{i=1}^{N_{\mathrm{DG}}}\left[\chi_{\mathrm{DG}, i}^{\mathrm{up}}(k)+\chi_{\mathrm{DG}, i}^{\mathrm{down}}(k)\right. \\
& \left.+\mathrm{OM}_{\mathrm{DG}, i} \delta_{\mathrm{DG}, i}(k) \Delta t\right]+C_{\text {total }}
\end{aligned}
$$

The three terms within the brackets are the startup cost, the shutdown cost, and the maintenance and management costs, respectively. The term $C_{\text {total }}$ is the sum of the energy storage cost, the fuel cost of the generators, and the penalty cost of load curtailment. $C_{\text {total }}$ is expressed as

$$
C_{\text {total }}=\sum_{k=1}^{T}\left[C_{\text {bess }}(k)+C_{\mathrm{DG}}(k)+C_{\text {cur }}(k)\right] \text {, }
$$

where $C_{\text {bess }}(k)$ is the ESS maintenance cost for the time interval $k\left(C_{\text {bess }}(k)=\left|P_{\text {bess }}(k)\right| \mathrm{OM}_{\text {bess }} \Delta t\right), C_{\mathrm{DG}}(k)$ is the fuel cost of the controllable generators $\left(C_{\mathrm{DG}}(k)=\right.$ $\left.\sum_{i=1}^{N_{\mathrm{DG}}} C_{\mathrm{DG}, i}(k)=\sum_{i=1}^{N_{\mathrm{DG}}}\left(a_{i} P_{\mathrm{DG}, i}^{2}(k)+b_{i} P_{\mathrm{DG}, i}(k)+c_{i}\right) \Delta t\right)$, and $C_{\text {cur }}(k)$ is the cost associated with the impact of load curtailment on user comfort, i.e., the penalty or compensation cost of load curtailment $\left(C_{\text {cur }}(k)=\alpha_{\text {cur }}(k) \beta_{\text {cur }}(k) \widetilde{P}_{\text {cur }}(k) \Delta t\right.$, where $\alpha_{\text {cur }, i}(k)$ reflects the preference of various users).

\section{E-TSRO Model for Energy Management and Solution Algorithm}

3.1. Description of Uncertain Parameters. The primary focus of this study is the uncertainty in two types of factors that affect islanded microgrid energy management, namely, the power output from renewable (wind and solar) energy sources and the power of loads (critical and curtailable loads). It is assumed that the uncertainties in these factors can be represented by intervals. Here, the predicted output power of solar energy, $\widetilde{P}_{\text {solar }}(k)$, for the time interval $k$ is used as an example. Then, $\widetilde{P}_{\text {solar }}(k) \in\left[\bar{P}_{\text {solar }}(k)\right.$ $\left.-\widehat{P}_{\text {solar }}(k), \bar{P}_{\text {solar }}(k)+\widehat{P}_{\text {solar }}(k)\right]$, where $\bar{P}_{\text {solar }}(k)$ and $\widetilde{P}_{\text {solar }}(k)$ are the predicted expected output power and the deviation from the expected value of solar energy over time interval $k$, respectively. Spatial and temporal fluctuations in the uncertainties are incorporated in the model using two adjustable budget-of-uncertainty (BoU) parameters, $\Gamma_{S}$ and $\Gamma_{T}$ [33], respectively, which reflect the risk preference of the decision-maker.

As all the constraints in the proposed model are linear, the worst-case scenario will inevitably happen at one of the vertices of the polyhedral uncertain set [33]. The uncertain parameters considered in this study are given below:

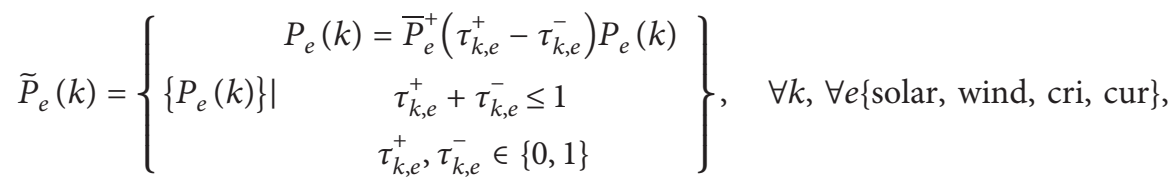

$$
\begin{aligned}
& \sum_{e \in\{\text { solar, wind, cri, cur }\}} \tau_{k, e}^{+}+\tau_{k, e}^{-} \leq \Gamma_{S}, \quad \forall k, \\
& \sum_{k} \tau_{k, e}^{+}+\tau_{k, e}^{-} \leq \Gamma_{T}, \quad \forall e \in\{\text { solar, wind, cri, cur }\} \\
& \tau_{k, e}^{+}, \tau_{k, e}^{-} \in\{0,1\}, \quad \forall k, \forall e \in\{\text { solar, wind, cri, cur }\}
\end{aligned}
$$

Considering that the uncertain parameters incorporated in this paper include the power of renewable energy (i.e., wind and solar) and the power of critical and curtailable loads, the probability that actual outputs of these uncertain parameters reach their upper or lower bound at the same time is very small. Therefore, a spatial constraint on the BoU 
parameter of $\Gamma_{S}$ is imposed denoting by equation (16). Similarly, equation (17) represents the temporal constraint on the BoU of $\Gamma_{T}$.

3.2. E-TSRO Model. The aforementioned uncertain parameters and their expressions were used to classify all the decision variables into decision variables for the day-ahead (prescheduling) stage and the intraday (rescheduling) stage. Considering limitations such as the startup and shutdown costs of DGs, the required durations before startup and shutdown, and the ramp power, only the on/off status variable of the DGs is treated as a day-ahead stage decision variable, i.e., the value of the on/off status variable must be determined a day ahead based on the predicted ranges of the uncertain parameters. The remaining decision contents are intraday or rescheduling decision variables. The E-TSRO model is expressed below [34]:

$$
\begin{aligned}
& \min _{x, y^{0}} c^{T} x+d^{T} y^{0}, \\
& \text { s.t. } \quad A x+B y^{0} \leq b-C U^{0}, \\
& \forall u \in U, \exists y: B y \leq b-C u-A x, \\
& x \in X, y^{0} \geq 0, y \geq 0,
\end{aligned}
$$

where $x$ and $y$ are first- and second-stage decision variables, respectively, $u$ is an uncertain parameter, and $y^{0}$ is the decision corresponding to $y$ when the value of the uncertain parameter $u$ is set to its predicted expected value $u^{0}$. Equation (19a) shows the operational cost of the system for minimization under the expected scenario. Equation (19b) is the constraint on the system under the expected scenario. Equation (19c) expresses the robust feasibility of the prescheduling solution $x$, i.e., for a given prescheduling strategy $x$, when the uncertain parameter $u$ is set to a value within the given range, there is a feasible solution for the second-stage decision variable $y$. Equations (19a)-(19d) show that, in the E-TSRO model, the optimization of the cost under the worst-case scenario in the C-STRO model is replaced by the optimization of the cost $d^{T} y^{0}$ under the expected scenario. The E-TSRO model also requires the prescheduling strategy to be feasible under any scenario. The change in the focus of the model from the worst-case scenario to the expected scenario results in a less conservative decision, while ensuring that a feasible decision can be obtained when knowledge of the uncertain parameter values becomes available. Thus, an optimal decision is ensured under the expected scenario and feasible under the worst-case scenario.

The correspondence between equations (19a)-(19d) and the microgrid energy management model presented in Section 2 is as follows: equation (19a) corresponds to equation (13); equation (19b) corresponds to equations (1)-(12), and the only difference being that the predicted range of the uncertain parameter is replaced by the predicted expected value; $B y \leq b-C u-A x$ in equation (19c) corresponds to replacing the predicted value of the uncertain parameter by its actual value in equations (1)-(12); for equation $(19 \mathrm{~d}), x$ represents $\delta_{D G}(k)$, and $y$ represents the remaining decision variables. However, equation (19c) makes it impossible to directly solve the model given by equations (19a)-(19d). Instead, a set of prescheduling solutions must be found, which satisfies robust feasibility, and a corresponding test model must be established. A method for establishing a test model based on equations (19a)-(19d) and a solution algorithm are presented below.

3.3. Robust Feasibility Test Model for Prescheduling Solutions. The method reported by $[33,35]$ for establishing a robust feasibility test model is used here to determine a set of prescheduling solutions that satisfy robust feasibility. First, the robust feasible region of the prescheduling variable $x$ denoted by equation (19c) is rewritten as follows:

$$
X_{R}=\{x \in X \mid \forall u \in U, Y(x, u) \neq \varnothing\},
$$

where $Y(x, u)=\{y \mid B y \leq b-C u-A x\}$ is a feasible solution to the rescheduling decision variable $y$ based on the given prescheduling variable $x$ and the uncertain parameter $u$. For the given $u \in U$, two positive slack variables, $s^{+}$and $s^{-}$, are introduced to test whether $Y(x, u)$ is null. These variables can be interpreted as emergency measures to prevent system failure. Accordingly, a linear programming problem is defined as follows:

$$
\begin{aligned}
& r\left(x^{*}, u\right)=\min _{y, s^{+}, s^{-}} 1^{T} s^{+}+1^{T} s^{-} \\
& \text {s.t. } \mathrm{By}+1^{T} s^{+}-1^{T} s^{-} \leq b-C u-A x^{*} .
\end{aligned}
$$

The objective function in equation (21) is to minimize the sum of the slack variables $s^{+}$and $s^{-}$based on the given prescheduling decision $x^{*}$ and knowledge of the value of the uncertain parameter $u$. $r\left(x^{*}, u\right)=0$ means $Y\left(x^{*}, u\right) \neq \varnothing$. Based on the model established in Section 2.2, the constraints corresponding to those in equation (21) are as follows:

$$
\left\{\begin{array}{l}
P_{\text {bess }}^{\min } \leq P_{\text {bess }}(k) \leq P_{\text {bess }}^{\max }, \quad \forall k, \\
E_{\text {bess }}^{\min } \leq E_{\text {bess }}(k) \leq E_{\text {bess }}^{\max }, \quad \forall k, \\
E_{\text {bess }}(k+1)=E_{\text {bess }}(k)+\eta_{\text {bess }} P_{\text {bess }}(k) \Delta t-\varepsilon_{\text {bess }} \Delta t, \quad \forall k, \\
-\Delta P_{\mathrm{DG}, i} \Delta t \leq P_{\mathrm{DG}, i}(k)-P_{\mathrm{DG}, i}(k-1) \leq \Delta P_{\mathrm{DG}, i} \Delta t, \quad \forall i, \forall k, \\
\delta_{\mathrm{DG}, i}^{*}(k) P_{\mathrm{DG}, i}^{\min } \leq P_{\mathrm{DG}, i}(k) \leq \delta_{\mathrm{DG}, i}^{*}(k) P_{\mathrm{DG}, i}^{\max }, \quad \forall i, \forall k, \\
\beta_{\text {cur }}^{\min } \leq \beta_{\mathrm{cur}}(k) \leq \beta_{\mathrm{cur}}^{\mathrm{max}}, \quad \forall i, \forall k, \\
P_{\text {cur }}(k)\left(1-\beta_{\text {cur }}(k)\right)+P_{\mathrm{cri}}(k)+\left(u_{\mathrm{bess}}(k)-v_{\text {bess }}(k)\right) \\
=P_{\text {solar }}(k)+\widetilde{P}_{\text {wind }}(k)+\sum_{i=1}^{N_{\mathrm{DG}}} P_{\mathrm{DG}, i}(k)+s_{k}^{+}-s_{k}^{-}, \quad \forall i, \forall k .
\end{array}\right.
$$

Based on the definition above, if $r\left(x^{*}, u\right)=0$, then $Y\left(x^{*}, u\right) \neq \varnothing$; otherwise, if $r\left(x^{*}, u\right)>0$, then $Y\left(x^{*}, u\right)=\varnothing$, which means a feasible rescheduling result cannot be obtained. To analyse the robustness of $x^{*}$, the result of equation 
(21) must be tested under the worst-case scenario based on the uncertainty in $u$. Thus, the following equation is defined based on equation (21):

$$
\begin{aligned}
& R\left(x^{*}\right)=\max _{u \in U} r\left(x^{*}, u\right)=\max _{u \in U} \min _{y, s^{+}, s^{-}} 1^{T} s^{+}+1^{T} s^{-} \\
& \text {s.t. } \quad \mathrm{By}+1^{T} s^{+}-1^{T} s^{-} \leq b-C u-A x^{*} .
\end{aligned}
$$

The uncertainty set $U$ is determined by equations (15)-(18). This max-min form is used to determine whether a feasible rescheduling decision can be obtained under the worst-case scenario based on the given prescheduling decision $x^{*}$, which can be addressed with KKT condition or dual theory [26]. As for equation (21), if $R\left(x^{*}\right)=0$, then $Y\left(x^{*}, u\right) \neq \varnothing$, and vice versa. As there is no integer variable in $y$, the max-min decision problem of equation (23) is dually transformed to the max bilinear programming problem shown below:

$$
R\left(x^{*}\right)=\max _{u \in U, w \in W} w^{T}(b-A x)-w^{T} C u,
$$

where $w$ is the dual of the variable in equation (24) and $W=\left\{w \mid w^{T} B \leq 0^{T},-1^{T} \leq w^{T} \leq 0^{T}\right\}$.

3.4. Algorithm for Solving the E-TSRO Model. Equation (23) shows that the prescheduling strategy is robust feasible only for $R\left(x^{*}\right)=0$ [33]. Otherwise, new constraints must be continually added to the main problem (MP) given by equations (19a) and (19b) until $R\left(x^{*}\right)=0$. Here, we use the $\mathrm{C} \& \mathrm{CG}$ algorithm to solve the model according to the main principle as follows. Based on the aforementioned two-stage classification, a set of prescheduling solutions is obtained by solving the MP given by equations (19a) and (19b); then, the robust feasibility test model given by equation (24) is solved using the obtained solutions. If $R\left(x^{*}\right)>0$, it means that no feasible solution can be obtained during the rescheduling stage, i.e., the power balance of the system cannot be achieved, and a constraint that corresponds to the scenario determined by solving equation (24) should be added to the MP. The MP is then solved again. Cyclic interactive iterations are performed until $R\left(x^{*}\right)=0$, which means a robust feasible prescheduling solution is found.

The steps of the C \& CG algorithm are outlined below, and the corresponding diagram is also illustrated as in Figure 1.

Step 1. Set the number of iterations $k=0, x^{0}=0$, and $R^{0}=0$.

Step 2. Solve the following MP:

$$
\begin{aligned}
& \min _{x, y^{0}} c^{T} x+d^{T} y^{0} \\
& \text { s.t. }\left\{\begin{array}{l}
A x+B y^{0} \leq b-C u^{0}, \\
A x+B y^{l} \leq b-C u_{l}^{*}, \quad 0 \leq l \leq k,
\end{array}\right.
\end{aligned}
$$

and denote the solution as $x_{k+1}^{*}$.

Step 3. Solve the subproblem shown in equation (24) with the obtained solution $x_{k+1}^{*}$. Denote the value of the second-stage variable $y$ by $y^{k+1}$, the determined value of $u$ by $u_{k+1}^{*}$, and the obtained value of the optimization objective by $R^{k+1}$. If $R^{k+1}=0$, take $x_{k+1}^{*}$ as a robust feasible optimal prescheduling solution and proceed to Step 4; otherwise, add the following constraint to the MP:

$$
A x+B y^{k+1} \leq b-C u_{k+1}^{*}
$$

Let $k=k+1$ and return to Step 2

Step 4. Solve the rescheduling model as follows:

$$
\begin{aligned}
& \min _{y} d^{T} y \\
& \text { s.t. } \quad \mathrm{By} \leq b-C u_{r}-A x_{k+1}^{*} \text {, }
\end{aligned}
$$

where $u_{r}$ is the actual value of $u$ and $x_{k+1}^{*}$ is the robust feasible optimal prescheduling solution obtained in Step 3.

\section{Experimental Validation and Analysis}

4.1. Experimental Environment and Parameter Settings. Simulations were performed on an islanded microgrid to analyse and validate the established E-TSRO model and the solution algorithm. The islanded microgrid contains three diesel generators, a battery ESS, PV and wind power generators, and critical and curtailable loads. The development, programming, and solution of the model, as well as the implementation of the rescheduling stage were built using IBM ILOG CPLEX V12.2. The model was used to perform day-ahead scheduling. The duration of energy scheduling was set to 24 hours that starts at 0:00 and ends at 24:00, and the unit time interval was set to 1 hour. The rated power of the wind and PV generators was set to $200 \mathrm{~kW}$ in order to make the total energy consumption and the energy supply of the system approximately balance within the setting 24 hours. Tables 4 and 5 summarize the relevant parameters of the DGs and ESS, respectively, and the parameter settings of diesel generators are mainly from literature [36].

Data for the wind, PV devices, and loads used in this study were obtained by integrating relevant data published by the Belgian Power Transmission Database in May 2018, as shown in Figure 2. The solid lines represent the predicted expected values of the uncertain parameters (wind power, solar power, and loads). For each time interval, the ratio of the critical loads to the curtailable loads in the system is $7: 3$. The areas enclosed by the dotted lines are the fluctuation ranges of the uncertainties. In addition, the prediction error percentage was set to increase linearly with time (from 3.5\% at the 1 st h to $15 \%$ at the 24 th $h$ at a rate of $0.5 \%$ per $h$ ), in order to reflect the trend that the prediction error normally increases over time.

Load curtailment can alleviate the energy supply burden when the energy supply is inadequate and is an indispensable adjustment measure for an islanded microgrid system. However, load curtailment may affect user comfort and is therefore generally not encouraged. As a result, the penalty for load curtailment should be set based on the actual situation. In this study, the proportion of curtailable 


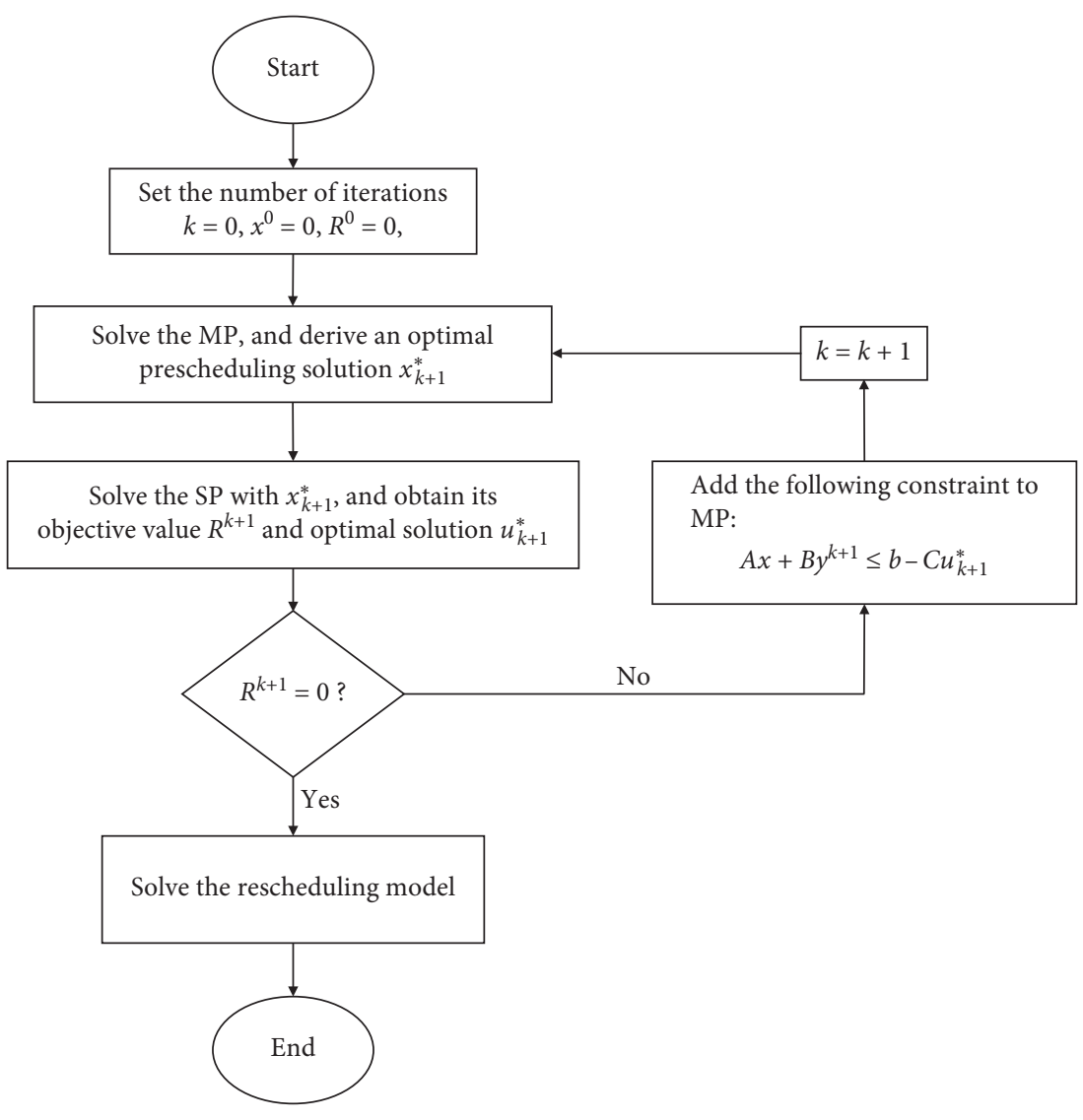

Figure 1: Diagram of the C \& CG algorithm.

TABle 4: Parameters of DGs.

\begin{tabular}{lcccccc}
\hline $\mathrm{DG}$ & $P_{\mathrm{DG}, i}^{\max } / P_{\mathrm{DG}, i}^{\min }(\mathrm{kW})$ & $\Delta P_{\mathrm{DG}, i}(\mathrm{~kW})$ & $T_{i}^{\mathrm{up}} / T_{i}^{\mathrm{down}}(\mathrm{h})$ & $a_{i} / b_{i} / c_{i}$ & $c_{\mathrm{DG}, i}^{\mathrm{up}} / c_{\mathrm{DG}, i}^{\mathrm{down}}(€)$ & $\mathrm{OM} \mathrm{DG}_{\mathrm{D}, i}(€ / \mathrm{h})$ \\
\hline 1 & $82 / 6.4$ & 60 & $2 / 2$ & $0.00421 / 0.2304 / 3.428$ & 2 & 0.5 \\
2 & $80 / 6$ & 60 & $4 / 4$ & $0.00209 / 0.2254 / 3.428$ & 3 & 0.9 \\
3 & $62 / 2.3$ & 50 & $2 / 2$ & $0.00303 / 0.2278 / 5.722$ & 3 \\
\hline
\end{tabular}

TABle 5: Parameters of ESS.

\begin{tabular}{lcccccc}
\hline$E_{\text {bess }}^{\max }(\mathrm{kW} \cdot \mathrm{h})$ & $E_{\text {bess }}^{\min }(\mathrm{kW} \cdot \mathrm{h})$ & Initial capacity $(\mathrm{kW} \cdot \mathrm{h})$ & $P_{\text {bess }}^{\max }(\mathrm{kW})$ & $\mathrm{OM}_{\text {bess }}(€ /(\mathrm{kW} \cdot \mathrm{h}))$ & $\eta_{\text {bess }}(\%)$ & $\varepsilon_{\text {bess }}(\mathrm{kW})$ \\
\hline 1000 & 400 & 700 & 100 & 0.08 & 100 & 0.02 \\
\hline
\end{tabular}

loads at a single time point was set to 0.2 . The power generation cost per unit of the DGs considered in the experiment is approximately from $0.4 € / \mathrm{kW}$ to $0.65 € / \mathrm{kW}$. Hence, the load curtailment cost was set to $1 € / \mathrm{kW}$, which is approximately twice the power generation cost per unit of the generators.

\subsection{Experimental Results and Analysis}

4.2.1. Impact of the BoUs on the System Behaviour. Equations (16) and (17) show that the prescheduling result is affected by the two BoUs used to describe an uncertain parameter, that is, $\Gamma_{T}$, which is related to the number of periods, and $\Gamma_{S}$, which is related to the number of uncertain parameters. Accordingly, the model was solved where $\Gamma_{T}$ is fixed as 24 and $\Gamma_{S}$ is set from 0 to 3 , respectively, where 0 means all uncertain parameters are deterministic and 3 denotes that the output of solar energy, wind energy, and user load are simultaneously uncertain. The results of on/off status of the three DGs are summarized in Table 6. Similarly, Table 7 summarizes the status for the three DGs with different settings of $\Gamma_{T}$ and a fixed $\Gamma_{S}$ setting of 3 .

Tables 6 and 7 show that, as $\Gamma_{S}$ or $\Gamma_{T}$ increases, there is an overall increase in the conservativeness of the prescheduling solution obtained from the model, as reflected in the increased number of operational DGs. In addition, changing the BoU settings does not necessarily result in changes to the 


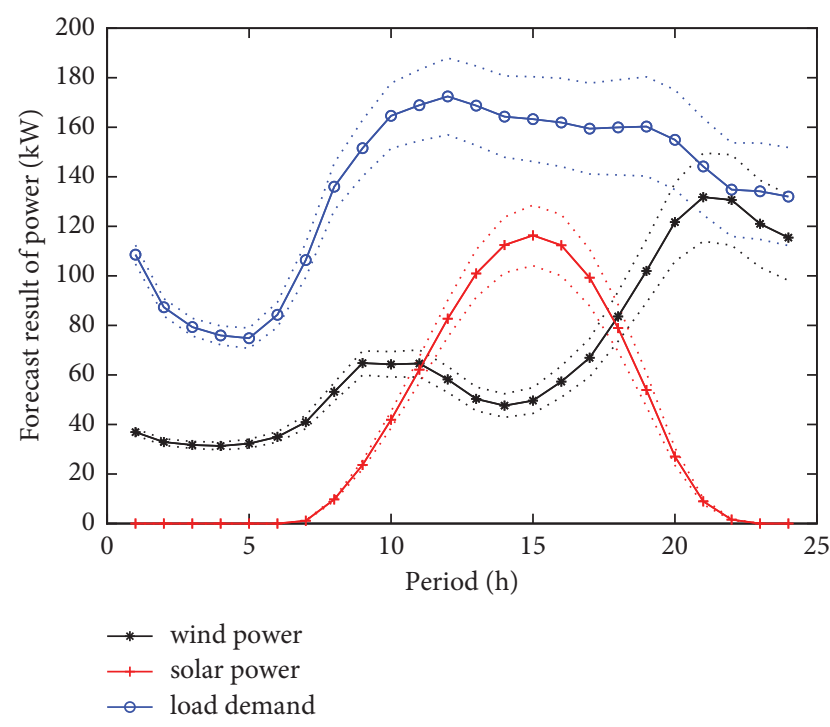

Figure 2: Predicted wind, solar, and load power.

TABLE 6: Prescheduling strategies for the status of DGs at various $\Gamma_{S}$ values $\left(\Gamma_{T}=24\right)$.

\begin{tabular}{lcccc}
\hline \multirow{2}{*}{ Period } & \multicolumn{4}{c}{ Strategies in predispatch stage } \\
& $\Gamma_{S}=0$ & $\Gamma_{S}=1$ & $\Gamma_{S}=2$ & $\Gamma_{S}=3$ \\
\hline 1 & $0 / 1 / 1$ & $0 / 1 / 1$ & $1 / 1 / 1$ & $1 / 1 / 1$ \\
2 & $0 / 1 / 1$ & $0 / 1 / 1$ & $1 / 1 / 1$ & $1 / 1 / 1$ \\
3 & $0 / 1 / 1$ & $0 / 1 / 1$ & $1 / 1 / 1$ & $1 / 1 / 1$ \\
4 & $0 / 1 / 1$ & $0 / 1 / 1$ & $1 / 1 / 1$ & $1 / 1 / 1$ \\
5 & $0 / 1 / 1$ & $0 / 1 / 1$ & $1 / 1 / 1$ & $1 / 1 / 1$ \\
6 & $0 / 1 / 1$ & $0 / 1 / 1$ & $1 / 1 / 1$ & $1 / 1 / 1$ \\
7 & $0 / 1 / 1$ & $0 / 1 / 1$ & $1 / 1 / 1$ & $1 / 1 / 1$ \\
8 & $0 / 1 / 1$ & $0 / 1 / 1$ & $1 / 1 / 1$ & $1 / 1 / 1$ \\
9 & $0 / 1 / 1$ & $0 / 1 / 1$ & $1 / 1 / 1$ & $1 / 1 / 1$ \\
10 & $0 / 1 / 1$ & $0 / 1 / 1$ & $1 / 1 / 1$ & $1 / 1 / 1$ \\
11 & $0 / 1 / 1$ & $0 / 1 / 1$ & $1 / 1 / 0$ & $1 / 1 / 0$ \\
12 & $0 / 0 / 1$ & $0 / 1 / 1$ & $1 / 1 / 0$ & $1 / 1 / 0$ \\
13 & $0 / 0 / 1$ & $0 / 1 / 0$ & $0 / 1 / 0$ & $0 / 1 / 0$ \\
14 & $0 / 0 / 1$ & $0 / 1 / 0$ & $0 / 1 / 0$ & $0 / 1 / 0$ \\
15 & $0 / 0 / 1$ & $0 / 0 / 0$ & $0 / 0 / 0$ & $0 / 0 / 0$ \\
16 & $0 / 0 / 0$ & $0 / 0 / 0$ & $0 / 0 / 0$ & $0 / 0 / 0$ \\
17 & $0 / 0 / 0$ & $0 / 0 / 0$ & $0 / 0 / 0$ & $0 / 0 / 0$ \\
18 & $0 / 0 / 0$ & $0 / 0 / 0$ & $0 / 0 / 0$ & $0 / 0 / 0$ \\
19 & $0 / 0 / 0$ & $0 / 0 / 0$ & $0 / 0 / 0$ & $0 / 0 / 0$ \\
20 & $0 / 0 / 0$ & $0 / 0 / 0$ & $0 / 0 / 0$ & $0 / 0 / 0$ \\
21 & $0 / 0 / 0$ & $0 / 0 / 0$ & $0 / 0 / 0$ & $0 / 0 / 0$ \\
22 & $0 / 0 / 0$ & $0 / 0 / 0$ & $0 / 0 / 0$ & $0 / 0 / 0$ \\
23 & $0 / 0 / 0$ & $0 / 0 / 0$ & $0 / 0 / 0$ & $0 / 0 / 0$ \\
24 & $0 / 0 / 0$ & $0 / 0 / 0$ & $0 / 0 / 0$ & $0 / 0 / 0$ \\
\hline & & & & \\
& & & &
\end{tabular}

status of DGs. For example, increasing $\Gamma_{S}$ from 2 to 3 (Table 6) and $\Gamma_{T}$ from 18 to 24 (Table 7) does not change the status of DGs. That is, the prescheduling results are clearly not overly sensitive to the changes in the BoUs.

To further analyse the impact of the BoUs on the rescheduling behaviour of the system, the following three special scenarios were defined according to the actual values assigned to the uncertain parameters at the second stage. (1) Expected scenario (Scenario A): each uncertain parameter is
TABLE 7: Prescheduling strategies for the status of DGs at various $\Gamma_{T}$ values $\left(\Gamma_{S}=3\right)$.

\begin{tabular}{lccccc}
\hline \multirow{2}{*}{ Period } & \multicolumn{5}{c}{ Strategies in predispatch stage } \\
& $\Gamma_{T}=0$ & $\Gamma_{T}=6$ & $\Gamma_{T}=12$ & $\Gamma_{T}=18$ & $\Gamma_{T}=24$ \\
\hline 1 & $0 / 1 / 1$ & $0 / 1 / 1$ & $1 / 1 / 1$ & $1 / 1 / 1$ & $1 / 1 / 1$ \\
2 & $0 / 1 / 1$ & $0 / 1 / 1$ & $1 / 1 / 1$ & $1 / 1 / 1$ & $1 / 1 / 1$ \\
3 & $0 / 1 / 1$ & $0 / 1 / 1$ & $1 / 1 / 1$ & $1 / 1 / 1$ & $1 / 1 / 1$ \\
4 & $0 / 1 / 1$ & $0 / 1 / 1$ & $1 / 1 / 1$ & $1 / 1 / 1$ & $1 / 1 / 1$ \\
5 & $0 / 1 / 1$ & $0 / 1 / 1$ & $1 / 1 / 1$ & $1 / 1 / 1$ & $1 / 1 / 1$ \\
6 & $0 / 1 / 1$ & $0 / 1 / 1$ & $1 / 1 / 1$ & $1 / 1 / 1$ & $1 / 1 / 1$ \\
7 & $0 / 1 / 1$ & $0 / 1 / 1$ & $1 / 1 / 1$ & $1 / 1 / 1$ & $1 / 1 / 1$ \\
8 & $0 / 1 / 1$ & $0 / 1 / 1$ & $1 / 1 / 1$ & $1 / 1 / 1$ & $1 / 1 / 1$ \\
9 & $0 / 1 / 1$ & $0 / 1 / 1$ & $1 / 1 / 1$ & $1 / 1 / 1$ & $1 / 1 / 1$ \\
10 & $0 / 1 / 1$ & $0 / 1 / 1$ & $1 / 1 / 1$ & $1 / 1 / 1$ & $1 / 1 / 1$ \\
11 & $0 / 1 / 1$ & $0 / 1 / 1$ & $1 / 1 / 0$ & $0 / 1 / 1$ & $0 / 1 / 1$ \\
12 & $0 / 0 / 1$ & $0 / 0 / 1$ & $1 / 1 / 0$ & $0 / 1 / 1$ & $0 / 1 / 1$ \\
13 & $0 / 0 / 1$ & $0 / 0 / 1$ & $0 / 1 / 0$ & $0 / 0 / 1$ & $0 / 0 / 1$ \\
14 & $0 / 0 / 1$ & $0 / 0 / 1$ & $0 / 1 / 0$ & $0 / 0 / 1$ & $0 / 0 / 1$ \\
15 & $0 / 0 / 1$ & $0 / 0 / 1$ & $0 / 1 / 0$ & $0 / 0 / 1$ & $0 / 0 / 1$ \\
16 & $0 / 0 / 0$ & $0 / 0 / 0$ & $0 / 0 / 0$ & $0 / 0 / 0$ & $0 / 0 / 0$ \\
17 & $0 / 0 / 0$ & $0 / 0 / 0$ & $0 / 0 / 0$ & $0 / 0 / 0$ & $0 / 0 / 0$ \\
18 & $0 / 0 / 0$ & $0 / 0 / 0$ & $0 / 0 / 0$ & $0 / 0 / 0$ & $0 / 0 / 0$ \\
19 & $0 / 0 / 0$ & $0 / 0 / 0$ & $0 / 0 / 0$ & $0 / 0 / 0$ & $0 / 0 / 0$ \\
20 & $0 / 0 / 0$ & $0 / 0 / 0$ & $0 / 0 / 0$ & $0 / 0 / 0$ & $0 / 0 / 0$ \\
21 & $0 / 0 / 0$ & $0 / 0 / 0$ & $0 / 0 / 0$ & $0 / 0 / 0$ & $0 / 0 / 0$ \\
22 & $0 / 0 / 0$ & $0 / 0 / 0$ & $0 / 0 / 0$ & $0 / 0 / 0$ & $0 / 0 / 0$ \\
23 & $0 / 0 / 0$ & $0 / 0 / 0$ & $0 / 0 / 0$ & $0 / 0 / 0$ & $0 / 0 / 0$ \\
24 & $0 / 0 / 0$ & $0 / 0 / 0$ & $0 / 0 / 0$ & $0 / 0 / 0$ & $0 / 0 / 0$ \\
\hline & & & & &
\end{tabular}

set to its predicted expected value. (2) Inadequate supply scenario (Scenario B): for each time interval, the wind and solar power are set to values to the lower bound of the predicted range, whereas the load demand is set to a value with the upper bound, denoting that a severely inadequate energy supply exists in this scenario. (3) Adequate supply scenario (Scenario C): for each time interval, the wind and solar power are set to values with the upper bound of the maximum predicted range, whereas the load demand is set with the lower bound. Tables 8 and 9 summarize the total cost of the system, the sum of the curtailed power, and the sum of the curtailed wind and PV power for the three typical scenarios under various BoU settings.

The following conclusions can be drawn from the results in Tables 8 and 9. (1) In Scenario A, the total cost of the system increases slightly (not significantly) with the BoUs. This finding is consistent with the impact of the BoUs on the system prescheduling result shown in Tables 6 and 7. (2) In Scenario B or C, at relatively low BoU values, no feasible rescheduling decision can be obtained for the system because as per the system requirements, the load curtailment ratio over each time interval cannot exceed 0.2 and wind and solar power curtailment is prohibited. Thus, for the given prescheduling decision, no rescheduling solution can be found that satisfies the power balance constraint on the system during the rescheduling stage, and this energy balance constraint can only be satisfied by increasing curtailment of the loads or wind and solar power. Analysing the relationship of the BoU settings to the total cost of the system, the total power of the curtailed loads, and the sum of the curtailed wind and solar power shows that increasing the 
TABLE 8: Rescheduling results for three typical scenarios at various $\Gamma_{S}$ values $\left(\Gamma_{T}=24\right)$.

\begin{tabular}{|c|c|c|c|c|c|c|c|c|c|}
\hline \multirow[b]{2}{*}{$\Gamma_{S}$} & \multicolumn{3}{|c|}{ Scenario A } & \multicolumn{3}{|c|}{ Scenario B } & \multicolumn{3}{|c|}{ Scenario C } \\
\hline & $\begin{array}{l}\text { Total } \\
\text { cost } \\
(€)\end{array}$ & $\begin{array}{c}\text { Total curtailed } \\
\text { power of loads } \\
(\mathrm{kW})\end{array}$ & $\begin{array}{c}\text { Total curtailed } \\
\text { power of } \\
\text { renewable energy } \\
(\mathrm{kW})\end{array}$ & $\begin{array}{c}\text { Total } \\
\text { cost }(€)\end{array}$ & $\begin{array}{c}\text { Total curtailed } \\
\text { power of loads } \\
(\mathrm{kW})\end{array}$ & $\begin{array}{c}\text { Total curtailed } \\
\text { power of } \\
\text { renewable energy } \\
(\mathrm{kW})\end{array}$ & $\begin{array}{c}\text { Total } \\
\text { cost }(€)\end{array}$ & $\begin{array}{l}\text { Total curtailed } \\
\text { power of loads } \\
\qquad(\mathrm{kW})\end{array}$ & $\begin{array}{c}\text { Total curtailed } \\
\text { power of } \\
\text { renewable energy } \\
(\mathrm{kW})\end{array}$ \\
\hline 0 & 546.1 & 0 & 0 & - & 103.1 & 0 & - & 0 & 80.6 \\
\hline 1 & 546.1 & 0 & 0 & - & 103.1 & 0 & - & 0 & 80.6 \\
\hline 2 & 546.6 & 0 & 0 & - & 103.1 & 0 & - & 0 & 8.4 \\
\hline 3 & 548.4 & 0 & 0 & 873.06 & 71.2 & 0 & 423.11 & 0 & 0 \\
\hline
\end{tabular}

TABLE 9: Rescheduling results for three typical scenarios at various $\Gamma_{T}$ values $\left(\Gamma_{S}=3\right)$.

\begin{tabular}{|c|c|c|c|c|c|c|c|c|c|}
\hline \multirow[b]{2}{*}{$\Gamma_{T}$} & \multicolumn{3}{|c|}{ Scenario A } & \multicolumn{3}{|c|}{ Scenario B } & \multicolumn{3}{|c|}{ Scenario C } \\
\hline & $\begin{array}{l}\text { Total } \\
\text { cost } \\
(€)\end{array}$ & $\begin{array}{l}\text { Total curtailed } \\
\text { power of loads } \\
(\mathrm{kW})\end{array}$ & $\begin{array}{c}\text { Total curtailed } \\
\text { power of } \\
\text { renewable energy } \\
(\mathrm{kW})\end{array}$ & $\begin{array}{c}\text { Total } \\
\text { cost }(€)\end{array}$ & $\begin{array}{l}\text { Total curtailed } \\
\text { power of loads } \\
\quad(\mathrm{kW})\end{array}$ & $\begin{array}{c}\text { Total curtailed } \\
\text { power of } \\
\text { renewable energy } \\
(\mathrm{kW})\end{array}$ & $\begin{array}{c}\text { Total } \\
\text { cost }(€)\end{array}$ & $\begin{array}{l}\text { Total curtailed } \\
\text { power of loads } \\
\quad(\mathrm{kW})\end{array}$ & $\begin{array}{c}\text { Total curtailed } \\
\text { power of } \\
\text { renewable energy } \\
(\mathrm{kW})\end{array}$ \\
\hline 0 & 546.1 & 0 & 0 & - & 103.1 & 0 & - & 0 & 80.6 \\
\hline 6 & 546.1 & 0 & 0 & - & 103.1 & 0 & - & 0 & 80.6 \\
\hline 12 & 546.9 & 0 & 0 & 869.7 & 71.2 & 0 & - & 0 & 67.3 \\
\hline 18 & 548.4 & 0 & 0 & 873.06 & 71.2 & 0 & 423.11 & 0 & 0 \\
\hline 24 & 548.4 & 0 & 0 & 873.06 & 71.2 & 0 & 423.11 & 0 & 0 \\
\hline
\end{tabular}

BoUs moderately increases the operational cost of the system and reduces load or wind and solar power curtailment in extreme cases. Thus, the system stability is moderately improved at an economic cost. (3) For the BoU settings that a solution can be obtained, a comparison of the three scenarios shows that the total system cost is highest under Scenario B (i.e., an extremely inadequate energy supply) and lowest under Scenario C (i.e., adequate energy supply). This result can be attributed to the large number of loads that must be curtailed in Scenario B, which results in a relatively high penalty cost, whereas the relatively low operational power of the generators in Scenario $\mathrm{C}$ results in a significant reduction of the fuel cost.

4.2.2. Analysis of the System Behaviour for Given BoUs. To determine the behaviour of typical devices (including the DGs and ESS) and users under given BoU settings, the BoUs were set to $\Gamma_{T}=24$ and $\Gamma_{T}=3$ using the aforementioned three scenarios as rescheduling scenarios, and the behaviour of the DGs, ESS, and curtailed loads for each scenario was recorded.

Figure 3(a) shows the power of the DGs and the proportion of curtailed loads for each time interval under the three typical scenarios. The prescheduling decision results in a consistent on/off status, but there is a notable difference in the total output power of the DGs among the three scenarios. As the energy supply in Scenario B is the most inadequate of the three scenarios by comparing the power generated from the renewable energy sources with the actual load demand, the generators maintain relatively high power during their on status periods. In contrast, the operational generators in Scenario C output relatively low power for their working periods. User loads need to be curtailed only in Scenario B because of the relative large inadequacy in the energy supply of the system. Figure 3(b) shows the ESS behaviour under the three scenarios. In Scenario B, to effectively respond to the inadequacy in the energy supply resulting from the shutdown of the generators in period 15, the ESS starts charging in cycle 0 until it is fully charged. The ESS starts discharging in cycle 15 to compensate for the energy supply deficit caused by the shutdown of the generators. In contrast, in Scenario C, the energy supply is relatively adequate. Thus, the ESS starts discharging in cycle 0 and shifts to discharge mode in cycle 15 to effectively utilize the remaining generation power after the wind power generators have started to supply power to the loads.

4.2.3. Comparison with the C-TSRO Model. Compared with the C-TSRO model, the most distinctive feature of the E-TSRO model is that it is oriented to the expected scenario instead of the worst-case scenario. Therefore, experiments were conducted to compare the economic efficiencies of the two models.

First, prescheduling decisions were obtained by solving both models with the setting of $\Gamma_{S}=3$ and $\Gamma_{T}=0,6,12,18$, and 24. Then, equation (27) was solved by assigning values to the uncertain parameters to obtain rescheduling decisions under the different scenarios. For example, under Scenario B and $\Gamma_{T}=12, \widetilde{P}_{\text {wind }}(k)$ was set to the lower bound of its predicted range for only 12 periods and to its expected value for the remaining periods. Table 10 is a comparison of the prescheduling and overall costs obtained by solving the two models for various $\Gamma_{T}$ values under the three rescheduling scenarios. 




(a)


(b)

FIgURE 3: System behaviour under three scenarios. (a) Behaviour of the DGs and load. (b) Behaviour of the ESS.

TABle 10: Comparison of costs obtained using two models under three classical scenarios for various $\Gamma_{T}$ values $\left(\Gamma_{S}=3\right)$.

\begin{tabular}{|c|c|c|c|c|c|c|c|c|c|c|c|c|}
\hline \multirow{3}{*}{$\Gamma_{T}$} & \multirow{2}{*}{\multicolumn{3}{|c|}{ Predispatch cost $(€)$}} & \multicolumn{9}{|c|}{ Total cost $(€)$} \\
\hline & & & & \multicolumn{3}{|c|}{ Scenario A } & \multicolumn{3}{|c|}{ Scenario B } & \multicolumn{3}{|c|}{ Scenario C } \\
\hline & C-TSRO & E-TSRO & CMPR (\%) & C-TSRO & E-TSRO & CMPR (\%) & C-TSRO & E-TSRO & CMPR (\%) & C-TSRO & E-TSRO & CMPR (\%) \\
\hline 0 & 33.9 & 33.9 & 0.0 & 546.2 & 546.2 & 0.0 & 546.2 & 546.2 & 0.0 & 546.2 & 546.2 & 0.0 \\
\hline 6 & 36.1 & 33.9 & 6.5 & 579.5 & 546.2 & 6.1 & 661.7 & 685.0 & -3.4 & 510.9 & 488.9 & 4.5 \\
\hline 12 & 46.5 & 43.5 & 6.9 & 583.5 & 546.9 & 6.7 & 765.1 & 797.8 & -4.1 & 491.1 & 466.4 & 5.3 \\
\hline 18 & 47.4 & 43.8 & 8.2 & 591.6 & 548.3 & 7.9 & 836.3 & 873.0 & -4.2 & 443.0 & 423.1 & 4.7 \\
\hline 24 & 47.7 & 43.8 & 8.9 & 593.8 & 548.3 & 8.3 & 832.8 & 873.0 & -4.6 & 446.8 & 423.1 & 5.6 \\
\hline
\end{tabular}

TABLE 11: Comparison of the average cost obtained using two models for various random values of uncertain parameters and different $\Gamma_{T}$ values $\left(\Gamma_{S}=3\right)$.

\begin{tabular}{|c|c|c|c|c|c|c|c|c|c|}
\hline \multirow{2}{*}{$\Gamma_{T}$} & \multicolumn{3}{|c|}{ Maximal total cost $(€)$} & \multicolumn{3}{|c|}{ Minimal total cost $(€)$} & \multicolumn{3}{|c|}{ Average total cost $(€)$} \\
\hline & C-TSRO & E-TSRO & CMPR (\%) & C-TSRO & E-TSRO & CMPR (\%) & C-TSRO & E-TSRO & CMPR (\%) \\
\hline 0 & 546.2 & 546.2 & 0.0 & 546.2 & 546.2 & 0.0 & 546.2 & 546.2 & 0.0 \\
\hline 6 & 633.8 & 617.0 & 2.7 & 517.9 & 501.5 & 3.3 & 587.6 & 551.2 & 6.6 \\
\hline 12 & 677.9 & 672.4 & 0.8 & 495.7 & 481.7 & 2.9 & 604.0 & 568.7 & 6.2 \\
\hline 18 & 740.9 & 717.1 & 3.3 & 475.3 & 444.4 & 7.0 & 632.2 & 593.6 & 6.5 \\
\hline 24 & 742.0 & 720.4 & 3.0 & 479.4 & 441.9 & 8.5 & 651.7 & 598.4 & 8.9 \\
\hline
\end{tabular}

The following interesting phenomena can be observed from Table 10. (1) Both models yield rescheduling solutions for $\Gamma_{T}=0$ and 6 because the values assigned to the uncertain parameters at the rescheduling stage were based on the actual value of $\Gamma_{T}$. In contrast, the results in Table 8 were obtained using a fixed $\Gamma_{T}=24$, resulting in no solutions under Scenarios B and C. (2) An analysis of the prescheduling costs in columns 2-4 of Table 10 shows that the C-TSRO model results in the generators being switched on/off a relatively large number of times and remaining operational over a relatively large number of periods. Therefore, the prescheduling cost from the C-TSRO model is higher (between 6.5-8.9\%) than that from the E-TSRO model. (3) A comparison of the total costs of the system 
shows that, under Scenarios A and C, the economic efficiency of the E-TSRO model is notably higher (between 4.5-8.3\%) than that of the C-TSRO model. However, under Scenario B (i.e., an extremely inadequate supply), the E-TSRO model does not offer any advantages over the C-TSRO model (from $-3.4 \%$ to $-4.6 \%$ ). This result is obtained because the C-TSRO model is oriented to the worstcase scenario, as represented by Scenario B. As a result, the generators are operational over more periods during the prescheduling stage compared with the solution of the E-TSRO model, preventing the curtailment of relevant loads at the rescheduling stage and the associated penalty effectively.

The economic efficiencies of the two models were further compared by using the aforementioned BoU settings to obtain prescheduling decisions. Thirty scenarios were randomly generated at the rescheduling stage, and the rescheduling models were solved under these 30 scenarios to calculate the total system cost under each scenario. Table 11 summarizes the maximum, minimum, and average costs for the 30 random scenarios, showing that the E-TSRO model is moderately more effective than the C-TSRO model. In addition, comprehensive statistical results show that the average total cost is reduced by about $6.7 \%$ based on different setting of $\Gamma_{T}$. The experiment also shows that the extreme case wherein load curtailment is required at the rescheduling stage does not occur under any of the 30 scenarios randomly generated at each $\Gamma_{T}$ value. This result further demonstrates that there is an infinitesimal probability of an extreme scenario occurring and underscores the necessity of responding to the impact of uncertain factors from an expected scenario perspective.

\section{Conclusions}

In this study, the uncertainties in factors such as power output from new (wind and solar) energy sources and user loads are addressed by dividing microgrid energy management decisions into day-ahead (prescheduling) stage decisions and real-time (rescheduling) stage decisions. These decisions are based on the operational characteristics and constraints of typical devices (e.g., generators) in a microgrid. This approach is moderately effective in reducing the impact of uncertain factors. An E-TSRO model for energy management is developed based on a typical islanded microgrid, using the aforementioned two decision-making stages and modelling the aforementioned uncertainties by ranges. Unlike the C-TSRO model, the E-TSRO model is oriented towards the expected scenario, while ensuring the feasibility of these prescheduling decisions under any realized scenario. As a result, the E-TSRO model can effectively improve the economic efficiency of the C-TSRO model at about $6.7 \%$. A C\&CG algorithm is developed to solve the E-TSRO model considering the model characteristics. The effectiveness of the E-TSRO model is validated and compared to that of the C-TSRO model through numerous experiments. The main conclusions are summarized as follows. (1) Although the BoU settings have some effect on the prescheduling decisions, this effect is not strictly linear, i.e., the prescheduling decision is not affected for some ranges of the BoU values. (2) Increasing the BoUs moderately increases the operational cost of the system. However, this increase also reduces load or wind and solar power curtailment in some extreme cases, thereby moderately improving the system stability. (3) In most of the investigated scenarios, the E-TSRO model is notably superior (i.e., from $4.5 \%$ to $8.9 \%$ ) to the C-TSRO model in terms of the economic efficiency of system operation, with the exception of the extreme scenario of a severely inadequate energy supply.

In this study, the charge efficiency coefficient is not distinguished from the discharge efficiency coefficient to simplify the dual transformation of the proposed model. In subsequent studies, a variable that characterizes the ESS charge/discharge status should be introduced to distinguish between these two coefficients. In addition, the E-TSRO model can also be analysed and validated by application to the energy management of grid-connected microgrids.

\section{Data Availability}

The data used to support the findings of this study are included within the article.

\section{Conflicts of Interest}

The authors declare that they have no conflicts of interest.

\section{Acknowledgments}

This work was supported by the funding of Coordinated Operation and Service Restoration for Interconnected Flexible AC/DC Urban Energy Systems under Grant no. 52061635104.

\section{References}

[1] M. F. Zia, E. Elbouchikhi, and M. Benbouzid, "Microgrids energy management systems: a critical review on methods, solutions, and prospects," Applied Energy, vol. 222, pp. 1033-1055, 2018.

[2] Z. Li and Y. Xu, "Temporally-coordinated optimal operation of a multi-energy microgrid under diverse uncertainties," Applied Energy, vol. 240, pp. 719-729, 2019.

[3] P. Teimourzadeh Baboli, M. Shahparasti, M. Parsa Moghaddam, M. R. Haghifam, and M. Mohamadian, "Energy management and operation modelling of hybrid AC-DC microgrid," IET Generation, Transmission \& Distribution, vol. 8, no. 10, pp. 1700-1711, 2014.

[4] S. Mohammadi, S. Soleymani, and B. Mozafari, "Scenariobased stochastic operation management of MicroGrid including wind, photovoltaic, micro-turbine, fuel cell and energy storage devices," International Journal of Electrical Power \& Energy Systems, vol. 54, pp. 525-535, 2014.

[5] W. Su, J. Wang, and J. Roh, "Stochastic energy scheduling in microgrids with intermittent renewable energy resources," IEEE Transactions on Smart Grid, vol. 5, no. 4, pp. 1876-1883, 2014.

[6] A. Ghasemi, "Coordination of pumped-storage unit and irrigation system with intermittent wind generation for 
intelligent energy management of an agricultural microgrid," Energy, vol. 142, pp. 1-13, 2018.

[7] J. Shen, C. Jiang, Y. Liu, and X. Wang, "A microgrid energy management system and risk management under an electricity market environment," IEEE Access, vol. 4, pp. 23492356, 2016.

[8] V. S. Tabar, M. A. Jirdehi, and R. Hemmati, "Energy management in microgrid based on the multi objective stochastic programming incorporating portable renewable energy resource as demand response option," Energy, vol. 118, pp. 827-839, 2017.

[9] M.-C. Hu, S.-Y. Lu, and Y.-H. Chen, "Stochastic programming and market equilibrium analysis of microgrids energy management systems," Energy, vol. 113, pp. 662-670, 2016.

[10] F. S. Gazijahani, S. N. Ravadanegh, and J. Salehi, "Stochastic multi-objective model for optimal energy exchange optimization of networked microgrids with presence of renewable generation under risk-based strategies," ISA Transactions, vol. 73, pp. 100-111, 2018.

[11] G. Cau, D. Cocco, M. Petrollese, S. Knudsen Kær, and C. Milan, "Energy management strategy based on short-term generation scheduling for a renewable microgrid using a hydrogen storage system," Energy Conversion and Management, vol. 87, pp. 820-831, 2014.

[12] N. Rezaei and M. Kalantar, "Stochastic frequency-security constrained energy and reserve management of an inverter interfaced islanded microgrid considering demand response programs," International Journal of Electrical Power \& Energy Systems, vol. 69, pp. 273-286, 2015.

[13] H. Farzin, M. Fotuhi-Firuzabad, and M. Moeini-Aghtaie, "Stochastic energy management of microgrids during unscheduled islanding period," IEEE Transactions on Industrial Informatics, vol. 13, no. 3, pp. 1079-1087, 2017.

[14] J. Liu, H. Chen, W. Zhang, B. Yurkovich, and G. Rizzoni, "Energy management problems under uncertainties for gridconnected microgrids: a chance constrained programming approach," IEEE Transactions on Smart Grid, vol. 8, no. 6, pp. 2585-2596, 2017.

[15] Z. Li and Y. Xu, "Optimal coordinated energy dispatch of a multi-energy microgrid in grid-connected and islanded modes," Applied Energy, vol. 210, pp. 974-986, 2018.

[16] D. Bertsimas and M. Sim, "The price of robustness," Operations Research, vol. 52, no. 1, pp. 35-53, 2004.

[17] R. Wang, P. Wang, and G. Xiao, "A robust optimization approach for energy generation scheduling in microgrids," Energy Conversion and Management, vol. 106, pp. 597-607, 2015.

[18] Z. Luo, W. Gu, Z. Wu, Z. Wang, and Y. Tang, "A robust optimization method for energy management of CCHP microgrid," Journal of Modern Power Systems and Clean Energy, vol. 6, no. 1, pp. 132-144, 2018.

[19] R. A. Gupta and N. K. Gupta, "A robust optimization based approach for microgrid operation in deregulated environment," Energy Conversion and Management, vol. 93, pp. 121-131, 2015.

[20] E. Kuznetsova, Y.-F. Li, C. Ruiz, and E. Zio, "An integrated framework of agent-based modelling and robust optimization for microgrid energy management," Applied Energy, vol. 129, pp. 70-88, 2014.

[21] Y. Zhang, N. Gatsis, and G. B. Giannakis, "Robust energy management for microgrids with high-penetration renewables," IEEE Transactions on Sustainable Energy, vol. 4, no. 4, pp. 944-953, 2013.
[22] Y. Xiang, J. Liu, and Y. Liu, "Robust energy management of microgrid with uncertain renewable generation and load," IEEE Transactions on Smart Grid, vol. 7, pp. 1034-1043, 2016.

[23] C. Zhang, Y. Xu, Z. Y. Dong, and K. P. Wong, "Robust coordination of distributed generation and price-based demand response in microgrids," IEEE Transactions on Smart Grid, vol. 9, no. 5, pp. 4236-4247, 2018.

[24] A. Gholami, T. Shekari, and S. Grijalva, "Proactive management of microgrids for resiliency enhancement: an adaptive robust approach," IEEE Transactions on Sustainable Energy, vol. 10, no. 1, pp. 470-480, 2019.

[25] Y. Guo and C. Zhao, "Islanding-aware robust energy management for microgrids," IEEE Transactions on Smart Grid, vol. 9, no. 2, pp. 1301-1309, 2018.

[26] B. Zeng and L. Zhao, "Solving two-stage robust optimization problems using a column-and-constraint generation method," Operations Research Letters, vol. 41, no. 5, pp. 457-461, 2013.

[27] W. Hu, P. Wang, and H. B. Gooi, “Toward optimal energy management of microgrids via robust two-stage optimization," IEEE Transactions on Smart Grid, vol. 9, no. 2, pp. 1161-1174, 2018.

[28] Y. Tian, L. Fan, Y. Tang, K. Wang, G. Li, and H. Wang, "A coordinated multi-time scale robust scheduling framework for isolated power system with ESU under high RES penetration," IEEE Access, vol. 6, pp. 9774-9784, 2018.

[29] B. Zhang, Q. Li, L. Wang, and W. Feng, "Robust optimization for energy transactions in multi-microgrids under uncertainty," Applied Energy, vol. 217, pp. 346-360, 2018.

[30] A. Hussain, V.-H. Bui, and H.-M. Kim, "Microgrids as a resilience resource and strategies used by microgrids for enhancing resilience," Applied Energy, vol. 240, pp. 56-72, 2019.

[31] D. A. Iancu and N. Trichakis, "Pareto efficiency in robust optimization," Management Science, vol. 60, no. 1, pp. 130-147, 2014.

[32] A. Parisio, E. Rikos, and L. Glielmo, "A model predictive control approach to microgrid operation optimization," IEEE Transactions on Control Systems Technology, vol. 22, no. 5, pp. 1813-1827, 2014.

[33] W. Wei, F. Liu, S. Mei, and Y. Hou, "Robust energy and reserve dispatch under variable renewable generation," IEEE Transactions on Smart Grid, vol. 6, no. 1, pp. 369-380, 2015.

[34] Y. An and B. Zeng, "Exploring the modeling capacity of twostage robust optimization: variants of robust unit commitment model," IEEE Transactions on Power Systems, vol. 30, no. 1, pp. 109-122, 2015.

[35] Z. Li, Y. Xu, S. Fang, X. Zheng, and X. Feng, "Robust coordination of a hybrid AC/DC multi-energy ship microgrid with flexible voyage and thermal loads," IEEE Transactions on Smart Grid, vol. 11, no. 4, pp. 2782-2793, 2020.

[36] A. Parisio and L. Glielmo, "A mixed integer linear formulation for microgrid economic scheduling," in Proceedings of the 2011 IEEE International Conference on Smart Grid Communications (SmartGridComm), pp. 505-510, Brussels, Belgium, October 2011. 